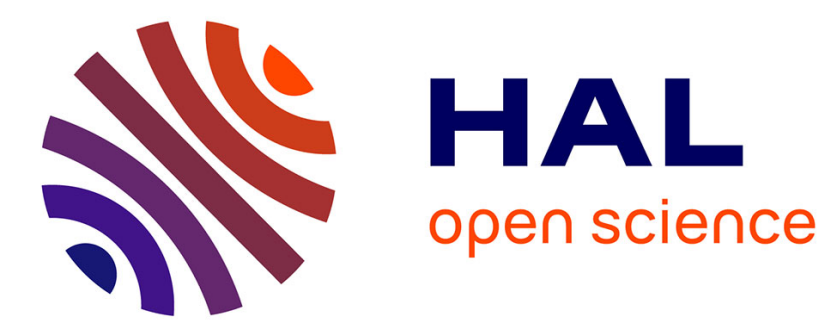

\title{
Average SIR Estimation in Cellular Networks with Best Server Policy
}

Mattia Minelli, Marceau Coupechoux, Jean-Marc Kélif

\section{To cite this version:}

Mattia Minelli, Marceau Coupechoux, Jean-Marc Kélif. Average SIR Estimation in Cellular Networks with Best Server Policy. IFIP Wireless Days, Oct 2010, Venice, Italy. pp.1-5. hal-01144496

\section{HAL Id: hal-01144496 https://hal-imt.archives-ouvertes.fr/hal-01144496}

Submitted on 21 Apr 2015

HAL is a multi-disciplinary open access archive for the deposit and dissemination of scientific research documents, whether they are published or not. The documents may come from teaching and research institutions in France or abroad, or from public or private research centers.
L'archive ouverte pluridisciplinaire HAL, est destinée au dépôt et à la diffusion de documents scientifiques de niveau recherche, publiés ou non, émanant des établissements d'enseignement et de recherche français ou étrangers, des laboratoires publics ou privés. 


\section{Average SIR Estimation in Cellular Networks with Best Server Policy}

\author{
Mattia Minelli \\ Télécom ParisTech and CNRS LTCI \\ 46, rue Barrault, Paris, France \\ mattia.minelli@enst.fr
}

\author{
Marceau Coupechoux \\ Télécom ParisTech and CNRS LTCI \\ 46, rue Barrault, Paris, France \\ coupecho@enst.fr
}

\author{
Jean-Marc Kelif \\ Orange Labs \\ Issy-Les-Moulineaux, France \\ jean-marc.kelif@orange-ftgroup.com
}

\begin{abstract}
- Abstract-The evaluation of the Signal to Interference Ratio (SIR) in cellular networks is of primary importance for network dimensioning. For static studies, which evaluate cell capacity and coverage, as well as for dynamic studies, which consider arrivals and departures of mobile stations (MS), the SIR is always an important input. Contrary to most of the analytical works evaluating SIR, we assume in this paper that the MS is attached to the best server, i.e., to the base station (BS) from which it receives the highest power. This is a policy that is more realistic than the classical assumption that considers MSs to be attached to the nearest BS. The exact formulation of the SIR is however in this case uneasy to handle and numerical methods remain heavy. In this paper, we thus propose an approximate analytical study based on truncated lognormal distributions that provides very close results to Monte Carlo simulations.
\end{abstract}

\section{INTRODUCTION}

Downlink capacity estimation is an issue of critical importance in cellular networks design, and it is directly bonded with the Signal over Interference Ratio (SIR) estimation.

Several papers address the capacity estimation matter under the assumption that the Mobile Station (MS) is always served by the nearest Base Station (BS), although the presence of shadowing in real mobile networks configures a more complex scenario: in fact, according to the most commonly used policy the MS is served by the station from which it receives more power (best server policy), which can be often different than the nearest BS. The shadowing effect, affecting each signal received by the MS and usually modeled as a lognormal random variable [9], depends on the local environment in which the MS is deployed and cannot be ignored while trying to model real situations. Moreover, the performance indicators analyzed in the papers are not ideal for the purpose of estimating the network capacity; for example, the OtherCell Interference Factor (OCIF), defined as the ratio of the total in-cell received power to the out-of-cell received power is often derived in place of the SIR.

In [4] and [5] the outage probability in presence of shadowing is derived without considering the best server, through the OCIF computation, while [13] and [12] compute the average OCIF (no best server) over a cell by numerical integration, considering a hexagonal network. In [2] the precise distribution of the other-cell interference is derived, although the derived formulas are quite difficult to be implemented.
The probability density function (PDF) and the cumulative density function (CDF) of the SIR in presence of fast fading and shadowing is found in [10], under the assumption that all the interferers have the same mean, which is unrealistic in real networks, where each BS has a different distance with respect to the MS. This assumption is not used in [11], but the formulae derived for the SIR distribution require the knowledge of the serving station, which is not given when the best server policy is used. [7] and [8] find the OCIF (no best server taken into account) for CDMA systems, using simulation and modeling.

In this paper, both the shadowing effect and the best server policy are taken into account to derive the average spatial SIR distribution in hexagonal networks. Section II introduces the system model, while in Section III the SIR expression is derived, making use of the probability for an MS to be served by a given station in the network, whose expression is shown in a dedicated subsection. An approximate method for the derivation of the average SIR, making use of the fluid network model [6] is then exposed in Section IV and validated in Section V through Monte-Carlo simulations, for a wide set of path-loss models and shadowing standard deviations.

\section{SYSTEM MODEL}

We consider the downlink of a hexagonal radio cellular network. All the BSs have omni-directional transmitting antennas and they transmit using the same power $P_{t x}$, while a frequency reuse 1 pattern is adopted, meaning that all the stations transmit on the same frequency. The BS density is $\rho_{B S}$, the half-distance between $\mathrm{BS}$ is $R_{c}$ and the cell range is $R$.

All the MSs are assumed to be served by the BS from which they receive more power, measured on the pilot signal, according to the so-called best server policy. MS location in the network can be measured with respect to its distance from the serving station (position in its logical cell, i.e., to which it is attached) or with respect to its distance from the nearest BS (position in the geographical cell). In this paper we always refer to the geographical cell because it represents a steady reference, while the logical cell changes according to the serving station.

The metric for the evaluation of the transmission quality is the average SIR $\gamma(d)$ experienced by an MS, expressed as a 
function of the distance $d$ from the nearest BS.

The propagation of radio signals is supposed to be affected by path-loss and lognormal shadowing, according to the following model:

$$
S_{i}(d)=P_{t x} K r_{i}^{-\eta} A,
$$

where $S_{i}(d)$ represents the received power from the $i$-th BS by an MS at a distance $d$ from the cell center, $K$ is a constant, $r_{i}$ is the distance between the considered MS and the $i$-th BS, $\eta$ is the path loss exponent and $A=10^{\frac{\xi}{10}}$ is a lognormal random variable (RV) taking into account the variations over the received power due to the shadowing effect. $\xi$ is a normal zero-mean $\mathrm{RV}$, whose standard deviation is denoted with $\sigma$ (in dB). Received power $S_{i}$ is thus a lognormal RV with PDF $f_{S_{i}}$ and $\operatorname{CDF} \Phi_{i}$. The background noise is not considered here, because its effects are neglectable with respect to the interference effect in urban environments.

The SIR is evaluated according to the position of a given MS in its cell, conventionally considered to be the 'central cell' of the network and covered by a BS conventionally named as 'BS1' (figure 1).

Let us divide the central cell into 6 sectors, each one covering an angle of $60^{\circ}$, having its vertex in the BS1, as shown in figure 1 . The borders of each sector are the lines joining the BS1 with its nearest neighbors BSs. Without any loss of generality, the generic MS we are considering in this paper can be assumed to be deployed in one of these sectors, thanks to the symmetries of the hexagonal network. As a further simplification, we assume MS to lie on the line splitting the chosen sector into two symmetric sectors of $30^{\circ}$ each (figure 1). This assumption doesn't meaningfully affect the validity of the obtained results, as shown in Section V.

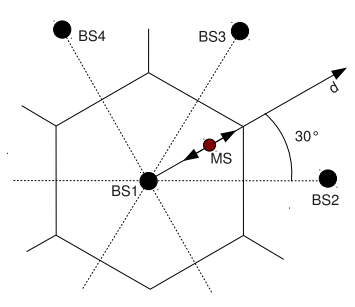

Fig. 1. System model

\section{SIR DERIVATION}

We are considering an MS being at a given distance $d$ from the cell center. For the sake of notation simplicity the index $d$ is omitted in the following.

In presence of shadowing, an MS could be served by any of the BSs. This makes the SIR computation very complicate in absence of further assumptions, because the serving BS choice strongly influences the experienced SIR. Let now suppose we know the probability $p_{i}$ for a given MS to be served by the $i$-th BS. We can compute the average SIR $\gamma_{i}$ knowing that the serving BS is BS $i$ and then $\gamma$ can be derived summing all the $\gamma_{i}$ for every possible serving station $i$, weighting each of them with its associated $p_{i}$ :

$$
\gamma=\sum_{i \in \mathcal{B}} \gamma_{i} p_{i}
$$

where $\mathcal{B}$ of cardinality $B$ is a set including all the indexes of the network BSs.

In Subsections III-A and III-B the expressions for $p_{i}$ and $\gamma_{i}$ are derived.

\section{A. Derivation of $p_{i}$}

We consider the quantity $L_{k}$, defined as the natural logarithm of the power $S_{k}$ received from the $k$-th BS:

$$
L_{k}=\ln \left(S_{k}\right)
$$

Given the lognormal distribution of $S_{k}, L_{k}$ is a normaldistributed RV, whose PDF $f_{L_{k}}$ is given by

$$
f_{L_{k}}(x)=\frac{1}{\sqrt{2 \pi a^{2} \sigma^{2}}} \exp \left(-\frac{\left(\ln (x)-\mu_{k}\right)^{2}}{2 a^{2} \sigma^{2}}\right),
$$

where $\mu_{k}=\ln \left(P_{t x} K r_{k}^{-\eta}\right)$ is the average value of $L_{k}$, and $a=\ln 10 / 10$.

The probability $p_{i}$ for an MS to be served by the $i$-th BS can be written as

$$
p_{i}=P\left(S_{i}>S_{h}, \quad \forall h \neq i\right)=P\left(L_{i}>L_{h}, \quad \forall h \neq i\right),
$$

which gives, taking into account the independence between all the $L_{k}$,

$$
\begin{aligned}
p_{i} & =\int_{-\infty}^{+\infty} P\left(L_{h}<x, \forall h \neq i\right) f_{L_{i}}(x) d x \\
& =\int_{-\infty}^{+\infty} \prod_{h \neq i} Q\left(\frac{\ln (x)-\mu_{h}}{a \sigma}\right) f_{L_{i}}(x) d x,
\end{aligned}
$$

where $Q(x)=1 / \sqrt{2 \pi} \int_{u}^{+\infty} e^{-u^{2} / 2} d u$ is the error function. Note that the computation of $p_{i}$ is very fast because few samples of the normal distribution are needed in order to have an accurate approximation of the integral, e.g. with the midpoint rule.

\section{B. Derivation of $\gamma_{i}$}

We introduce the random variables $\widetilde{S}_{h, i}$, which represent the received powers from $\mathrm{BSs} h \neq i$ knowing that $\mathrm{BS} i$ is the serving BS:

$$
\widetilde{S}_{h, i}=\frac{S_{h} \mathbf{1}_{\left\{S_{z}<S_{i}, \forall z \neq i\right\}}}{p_{i}} .
$$

We also define the interference power knowing that BS $i$ is the serving BS:

$$
\widetilde{I}_{i}=\sum_{h \neq i} \widetilde{S}_{h, i}
$$

With these notations, $\gamma_{i}$ can be written:

$$
\begin{aligned}
\gamma_{i} & =E\left[\frac{\widetilde{S}_{i, i}}{\widetilde{I}_{i}}\right] \\
& =\int_{0}^{+\infty} x E\left[\frac{1}{\widetilde{I}_{i}} \mid \widetilde{S}_{i, i}=x\right] f_{\widetilde{S}_{i, i}}(x) d x
\end{aligned}
$$


where $f_{\widetilde{S}_{i, i}}(x)$ is the PDF of the power received by the serving station.

On the one hand, this function can be obtained considering that

$$
\begin{aligned}
f_{\widetilde{S}_{i, i}}(x) d x & =P\left(S_{i}=x \mid S_{i}>S_{h}, \forall h \neq i\right) d x \\
& =P\left(S_{i}>S_{h}, \forall h \neq i \mid S_{i}=x\right) \frac{P\left(S_{i}=x\right) d x}{\left.p_{i}\right)} \\
& =\prod_{h \neq i} \Phi_{h}(x) \frac{f_{s_{i}}(x)}{p_{i}} d x
\end{aligned}
$$

where we exploited the fact that RVs $S_{h}$ are independent.

On the other hand, the expectation in (10) can be written as

$$
E\left[\frac{1}{\widetilde{I}_{i}} \mid \widetilde{S}_{i, i}=x\right]=\int \frac{1}{t} f_{\widetilde{I}_{i} \mid \widetilde{S}_{i, i}=x} d t .
$$

The function $f_{\widetilde{I}_{i} \mid \widetilde{S}_{i, i}=x}$ is the PDF of a sum of $B-1$ lognormal RVs truncated in $x$ :

$$
f_{\widetilde{I}_{i} \mid \widetilde{S}_{i, i}=x}=f_{\hat{S}_{1, x}} * \cdots * f_{\hat{S}_{i-1, x}} * f_{\hat{S}_{i+1, x}} * \cdots * f_{\hat{S}_{B, x}},
$$

where $*$ is the convolution operator and

$$
f_{\hat{S}_{h, x}}(y)=\frac{f_{S_{h}}(y)}{P\left(S_{h}<x\right)}, 0<y<x, h \neq i .
$$

We finally rewrite (10) as

$$
\begin{aligned}
\gamma_{i} & =\iint_{0}^{+\infty} \frac{x}{t} f_{\widetilde{S}_{i, i}}(x) f_{\widetilde{I}_{i} \mid \widetilde{S}_{i, i}=x}(t) d x d t \\
& =\iint_{0}^{+\infty} \frac{x}{t} \underset{\substack{* \\
h \neq i}}{*} f_{S_{h}}(t) \frac{f_{S_{i}}(x)}{p_{i}} d x d t .
\end{aligned}
$$

We now observe that $p_{i}$ can be simplified in equation (2):

$$
\gamma=\sum_{i \in \mathcal{B}} \iint_{0}^{+\infty} \frac{x}{t} \underset{\substack{* \\ h \neq i}}{ } f_{S_{h}}(t) f_{S_{i}}(x) d x d t .
$$

It is now theoretically possible to compute the average SIR using this formula. However, the convolution over the whole set of BS can be difficult to be performed in a large network. In the same way, infinite integrals computations are not easily performed for practical shadowing standard deviations because of the support of a lognormal PDF. Several approximations are however possible and give valid results, as shown in the next section.

\section{APPROXIMATE METHOD FOR $\gamma$ USING TRUNCATED LOGNORMALS}

We derive here an approximate method, valid on a wide range of values of $\sigma$ and $\eta$, which makes the computation of $\gamma_{i}$ sensibly simpler.

Let divide the set $\mathcal{B}$ in 2 sets, $\mathcal{B}=\mathcal{B}_{n} \cup \mathcal{B}_{f}$, where $\mathcal{B}_{n}$ of cardinality $N$ is the set of the indexes of the $N$ nearest $\mathrm{BSs}$, with respect to the central cell, while $\mathcal{B}_{f}$ of cardinality $B-N$ includes the indexes of all the other BSs in the network. According to (2), $\gamma_{i}$ is obtained as the sum of $B$ terms, where $B$ is the number of BS in the network and is thus potentially infinite. However, for all the practical values of $\eta$, the probabilities $p_{i}$ are meaningful only for the nearest BSs, whose indexes are included in $\mathcal{B}_{n}$. Thus we can write that

$$
\gamma \approx \sum_{i \in \mathcal{B}_{n}} \gamma_{i} p_{i}
$$

Under the condition that $\sigma$ is reasonably low and thanks to the delta method [3], we can approximate in our context $E\left[1 / \widetilde{I}_{i}\right]$ by $1 / E\left[\widetilde{I}_{i}\right]$ (the validation is done by simulations in Section V). The expression of $\gamma_{i}$ thus becomes: $\gamma_{i} \approx$ $E\left[\widetilde{S}_{i, i}\right] / E\left[\widetilde{I}_{i}\right]$. We now focus on each of these expectations by approximating involved RVs by truncated lognormal RVs.

\section{A. Average received signal}

The average power $E\left[\widetilde{S}_{i, i}\right]$ received by the serving BS is given by

$$
E\left[\widetilde{S}_{i, i}\right]=\int_{0}^{+\infty} x f_{\widetilde{S}_{i, i}}(x) d x,
$$

but the computation of the integral in (19) can be avoided. This average value can indeed be written:

$$
E\left[\widetilde{S}_{i, i}\right]=E\left[S_{i} \mid S_{h}<S_{i}, \forall h \neq i\right]
$$

Now, $S_{i}$ is lognormal RV, which is independent on all $S_{h}$ and is greater than all $S_{h}$ with probability $p_{i}$. We make the simple approximation that this happens only for the highest values of $S_{i}$. More precisely, we consider $S_{i}$ to be the maximum of all received signals when it is greater than some cut off value $\bar{r}_{i}$ such that $P\left(S_{i}>\bar{r}_{i}\right)=p_{i}$, i.e., $\bar{r}_{i}=\Phi_{i}^{-1}\left(1-p_{i}\right)$. It is illustrated on figure (2), where we assume that, above the threshold, $S_{i}$ is always the highest signal. As a consequence:

$$
\begin{aligned}
E\left[\widetilde{S}_{i, i}\right] & \approx E\left[S_{i} \mid S_{i}>\bar{r}_{i}\right] \\
& =e^{\mu_{i}+a^{2} \sigma^{2} / 2} \frac{1-\phi\left(\frac{\ln \left(\bar{r}_{i}\right)-\mu_{i}-a^{2} \sigma^{2}}{a \sigma}\right)}{1-\phi\left(\frac{\ln \left(\bar{r}_{i}\right)-\mu_{i}}{a \sigma}\right)},
\end{aligned}
$$

which is the average value of a truncated lognormal RV [1] and where $\phi$ is the standard normal CDF.

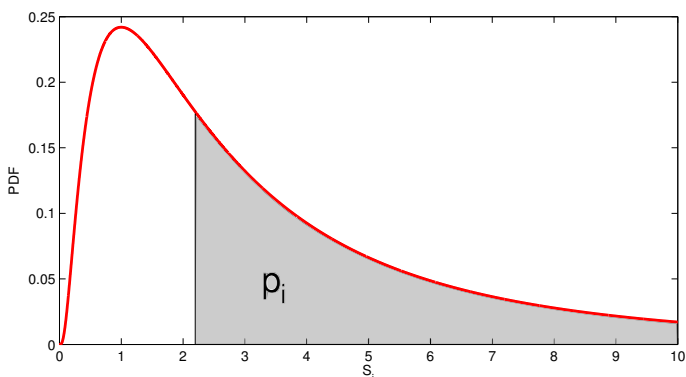

Fig. 2. Truncated lognormal. 


\section{B. Average interference}

Let us now consider the average interference. $E\left[\tilde{I}_{i}\right]$ takes into account the average interference seen by an MS served by the $i$-th station. We compute it as the sum of two terms, one taking into account the contribution of the far BSs (included in $\mathcal{B}_{f}$ ) and one taking into account the contribution of the near BSs (included in $\mathcal{B}_{n}$ ). Only the PDF of the power received by the BSs belonging to $\mathcal{B}_{n}$ are considered to be meaningfully modified with respect to the lognormal distribution, when the serving station is known. Thus the contribution $I_{f}$ of the far BSs can be approximated with the average of a sum of lognormal RV:

$$
\begin{aligned}
E\left[\tilde{I}_{i}\right] & \approx E\left[\sum_{h \in \mathcal{B}_{n}} \widetilde{S}_{h, i}\right]+E\left[\sum_{h \in \mathcal{B}_{f}} S_{h}\right] \\
& =\sum_{h \in \mathcal{B}_{n}} E\left[S_{h} \mid S_{i}>S_{h}, \forall h \neq i\right]+I_{f} .
\end{aligned}
$$

The precision of the approximation is determined by $N$.

The term $I_{f}=\sum_{h \in \mathcal{B}_{f}} E\left[S_{h}\right]$ can be computed starting from the expression found in [6], which well approximates the extra-cell received power without shadowing for best server policy, using the fluid model. Following this approach, taking into account the shadowing effect and removing the influence of the nearest BSs, we obtain:

$$
\begin{aligned}
I_{f} & =\int_{2 R_{c}-d}^{+\infty} \int_{0}^{2 \pi} \rho_{B S} P_{t x} K r^{-\eta} A r d r d \theta-E\left[\sum_{h \in \mathcal{B}_{n}} S_{h}\right] \\
& =\frac{2 \pi \rho_{B S} P_{t x} K}{\eta-2}\left[\left(2 R_{c}-d\right)^{2-\eta}\right] e^{\frac{a^{2} \sigma^{2}}{2}} \\
& -e^{\frac{a^{2} \sigma^{2}}{2}} \sum_{h \in \mathcal{B}_{n}} e^{\mu_{h}}
\end{aligned}
$$

Concerning the first term of the sum (22), we adopt the same approach as for the average received power. The distributions of the $\widetilde{S}_{h, i}$ are approximated by truncated lognormal distributions. In this case, we look for a common upper limit $\bar{r}_{i}^{\prime}$ for all interference received powers such that:

$$
\begin{aligned}
E\left[\widetilde{S}_{h, i}\right] & =E\left[S_{h} \mid S_{i}>S_{h}, \forall h \neq i\right] \\
& \approx E\left[S_{h} \mid S_{h}<\bar{r}_{i}^{\prime}\right] \\
& =e^{\mu_{i}+a^{2} \sigma^{2} / 2} \frac{\phi\left(\frac{\ln \left(\bar{r}_{i}^{\prime}\right)-\mu_{i}-a^{2} \sigma^{2}}{a \sigma}\right)}{\phi\left(\frac{\ln \left(\bar{r}_{i}^{\prime}\right)-\mu_{i}}{a \sigma}\right)} .
\end{aligned}
$$

By analogy with $\bar{r}_{i}$, we propose the following cut off value:

$$
\bar{r}_{i}^{\prime}=1-\Phi_{i}^{-1}\left(1-p_{i} / 2\right) .
$$

Simulations in Section V shows that this value provides a very good approximation.

Summarizing, the approximate method requires, for every average SIR value we want to estimate, the calculation of few formulas to evaluate the contribution of each of the $N$ terms considered in the derivation of $\gamma$. These operations include the computation of the average signal (according to formula (21)), the computation of $I_{f}$ (applying formula (24)), and the computation of the $N$ approximate values of $E\left[\widetilde{S}_{h, i}\right]$ (each derived as in formula (25)). All the computationally inconvenient integrals over the support of a lognormal RV are in this way avoided.

\section{RESULTS}

In this section, we compare our method to Monte Carlo simulations. All Monte-Carlo simulations are carried out considering a four rings hexagonal network (with range $R=$ $1 \mathrm{Km}$ ) with wraparound. At each snapshot, a MS dropped randomly with uniform spatial distribution in each sector. SIR is computed and averaged at a distance $d$ from the BS over a ring of ranges $d$ and $d+\delta(\delta=50 \mathrm{~m})$. Note that distance $d$ is the distance between MS and the BS of its geographical cell (nearest BS) although SIR is computed with the best server policy. Simulations are performed with 10000 snapshots.

\section{A. Simulation vs. Analysis}

Figures (3) and (4) show the comparison between the analytical approximation and the simulation results for different figures of the path-loss exponent $\eta$ and the shadowing standard deviation $\sigma$. In all cases, the matching is very good, even for $\sigma=10 \mathrm{~dB}$. It is interesting to see that the average SIR is increasing with $\sigma$ when the best server policy is chosen. This can be explained by the increased opportunity for a MS to receive a good signal from one of the neighbor BSs.

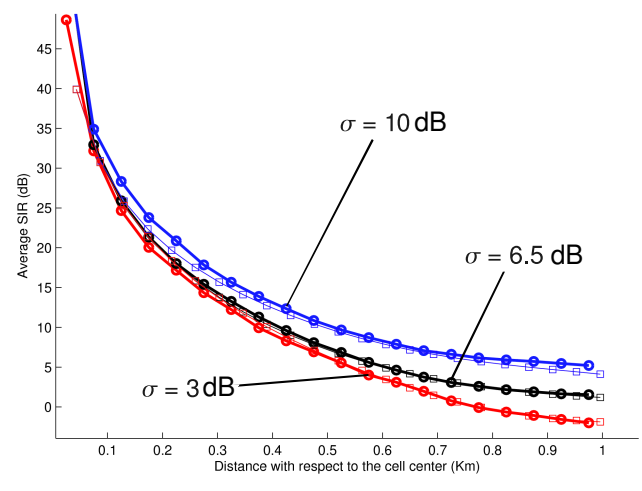

Fig. 3. SIR vs. distance to BS, approximate analysis (solid lines with circles) vs. simulations (solid lines with squares) $(\eta=3, N=18)$.

\section{B. Approximation Accuracy}

Figure (5) shows the improvement of the results accuracy while increasing the number $N$ of BSs considered as 'near' (in the set $\mathcal{B}_{n}$ ). The value of $N$ can be increased or decreased according to the desired trade-off between computational speed and precision of the results. The higher is $\sigma$, the higher should be $N$ for a given accuracy because the high variations of the received power increase the influence of farer BSs.

Figure (6) shows the accuracy of the approximations done for the computation of the average received signal (right) and of the average interference (left). On the y axis, we plot the difference between the simulated value and the approximation obtained with the truncated lognormal: $10 \log _{10} \mid E\left[\widetilde{S}_{i}\right]-$ 


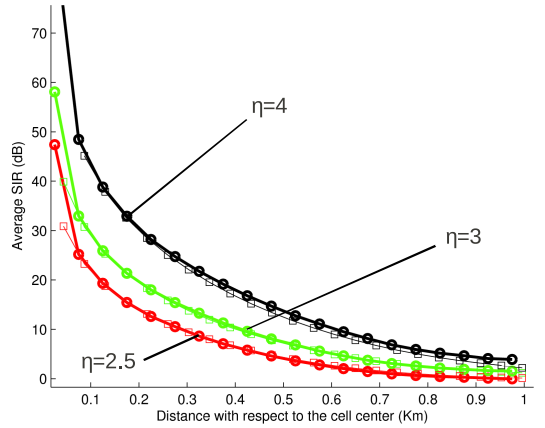

Fig. 4. SIR vs. distance to BS, approximate analysis (solid lines with circles) vs. simulations (solid lines with squares) $(\sigma=6.5 \mathrm{~dB}, N=18)$.

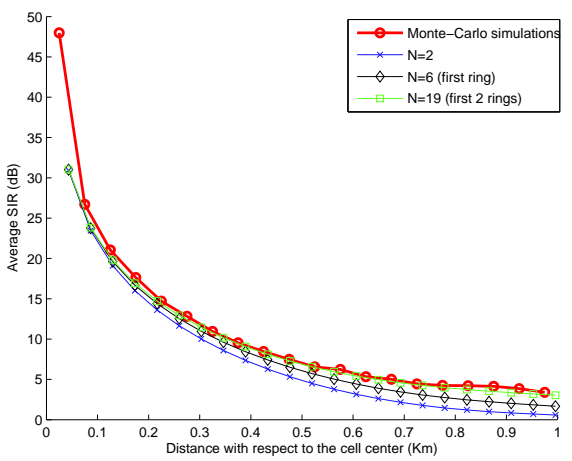

Fig. 5. SIR vs. distance to BS, influence of $N(\eta=2.5, \sigma=10 \mathrm{~dB})$.

$E\left[S_{i} \mid S_{i}>\bar{r}_{i}\right] \mid$ as a function of the cut off value $\bar{r}_{i}$ in logarithmic scale. The vertical line corresponds to $\bar{r}_{i}=\Phi_{i}^{-1}\left(1-p_{i}\right)$. In the same way, we plot $10 \log _{10}\left|\sum\left(E\left[\widetilde{S}_{h, i}\right]-E\left[S_{h} \mid S_{h}<\bar{r}_{i}^{\prime}\right]\right)\right|$ as a function of $\bar{r}_{i}^{\prime}$. The vertical line corresponds to $\bar{r}_{i}^{\prime}=$ $1-\Phi_{i}^{-1}\left(1-p_{i} / 2\right)$. In both cases, the induced error is very low and our cut off values are nearly optimal. Errors have been plotted for specific figures of $\eta, \sigma$ and $d$ but the same conclusion can be drawn for a wide range of realistic values of these parameters.
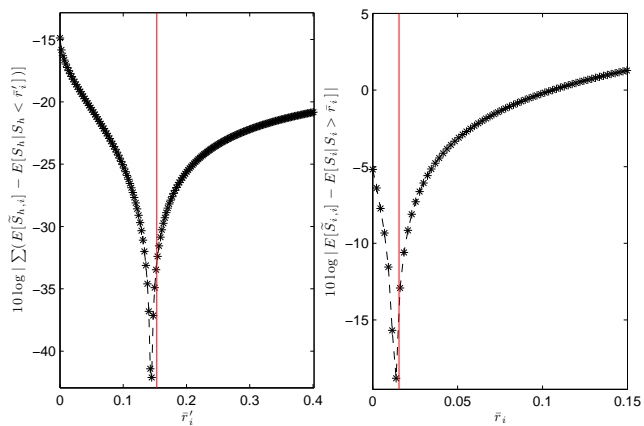

Fig. 6. Truncated lognormal approximation error vs. cut off value for average received power (right) and interference (left) $(\eta=4, \sigma=10 \mathrm{~dB}, d=$ $0.5 \mathrm{Km})$.

\section{Best Server vs. Non Best Server}

Figure (7) shows the influence of the best server policy. Average SIR is compared for the best server policy and for the nearest server policy as a function of the distance to the nearest BS. Until $d=500 \mathrm{~m}$, the nearest BS is also the best one with high probability. At cell border, the best server policy provides up to $4.3 \mathrm{~dB}$ gain.

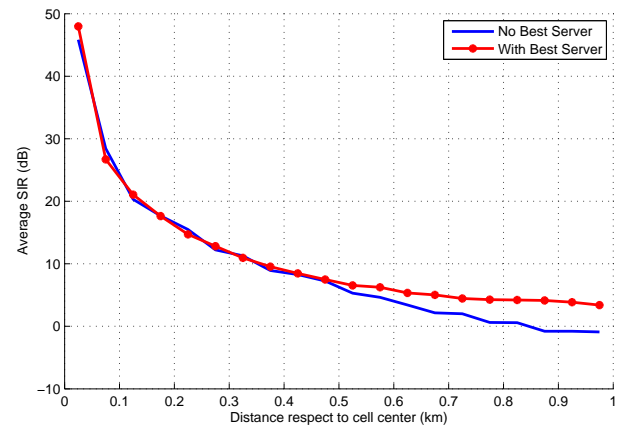

Fig. 7. SIR vs. distance to BS, influence of the MS attachment policy (best server vs. non best server) $(\eta=2.5, \sigma=10 \mathrm{~dB})$.

\section{CONCLUSION}

In this paper, we have proposed an efficient and fast analytical approximation for the estimation of the SIR in cellular networks with the realistic best server policy. This method is based on truncated lognormal random variables. Results show that the analytical approach gives very good results compared to Monte Carlo simulations.

\section{REFERENCES}

[1] I. Bebu and T.Mathew. Confidence intervals for limited moments and truncated moments in normal and lognormal models. Statistics and Probability Letters, 2009.

[2] C.C. Chan and S.V. Hanly. Calculating the outage probability in cdma network with spatial poisson traffic. IEEE Trans. on Vehicular Technology, Jan 2001.

[3] A. C. Davison. Statistical Models. Cambridge University Press, 2003.

[4] J.M. Kelif and M. Coupechoux. Effect of shadowing on outage probability in fluid cellular radio networks. WiOPT'08, 2008.

[5] J.M Kelif and M. Coupechoux. Impact of topology and shadowing on the outage probability of cellular networks. ICC'09, Jun 2009.

[6] J.M Kelif, M. Coupechoux, and P. Godlewski. Spatial outage probability for cellular networks. GLOBECOM '07, Nov 2007.

[7] S.S. Kolahi. Other cells interferences of power controlled cdma systems. IEEE International Conference on Personal Wireless Communicatons, Dec 2002.

[8] S.S. Kolahi, A.G. Williamson, and K.W. Sowerby. Other-cell interference in cdma systems. Electronics letters, Sep 2004.

[9] G.L. Stuber. Principles of mobile communications. Norwell, 1996.

[10] T.T. Tjhung and C.C. Chai. Distribution of sir and ber performance of ds-cdma systems in lognormally shadowed rician channels. IEEE Fifth International Symposium on Spread Spectrum Techniques and Applications, 1998.

[11] T.T. Tjhung and C.C. Chai. Distribution of sir and performance of dscdma systems in lognormally shadowed rician channels. IEEE Trans. on vehicular technology, Jul 2000.

[12] A.J. Viterbi. Cdma - principles of spread spectrum communication. Addison-Wesley, 1995.

[13] A.J. Viterbi, A.M. Viterbi, and E. Zehavi. Other-cell interference in cellular power-controlled cdma. IEEE Trans. on Communications, Feb/Mar/Apr 1994. 